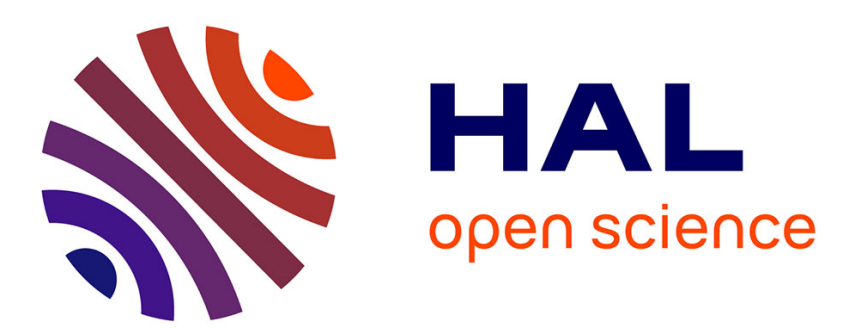

\title{
Des sociétés féminines de secours mutuels aux premières organisations féminines politisées en Guadeloupe et en Martinique au début du XXe siècle
}

\author{
Clara Palmiste
}

\section{- To cite this version: \\ Clara Palmiste. Des sociétés féminines de secours mutuels aux premières organisations féminines politisées en Guadeloupe et en Martinique au début du XXe siècle . Bulletin de la Société d'histoire de la Guadeloupe, 2009, 154, pp.79-92. 10.7202/1036849ar . hal-01672983}

\section{HAL Id: hal-01672983 \\ https://hal.univ-antilles.fr/hal-01672983}

Submitted on 14 Feb 2018

HAL is a multi-disciplinary open access archive for the deposit and dissemination of scientific research documents, whether they are published or not. The documents may come from teaching and research institutions in France or abroad, or from public or private research centers.
L'archive ouverte pluridisciplinaire HAL, est destinée au dépôt et à la diffusion de documents scientifiques de niveau recherche, publiés ou non, émanant des établissements d'enseignement et de recherche français ou étrangers, des laboratoires publics ou privés.

$$
\text { Copyright }
$$




\section{Des sociétés féminines de secours mutuel aux premières organisations féminines politisées en Guadeloupe et en Martinique au début du XXe siècle}

Clara Palmiste, Université des Antilles Guyane

[Publié dans le Bulletin de la Société d’Histoire de la Guadeloupe, n¹54, septembre-décembre 2009.]

\section{Introduction}

Par rapport aux discours et aux pratiques qui justifiaient l'exclusion des femmes de la sphère politique, il apparaît fondamental de comprendre comment les femmes des colonies françaises d'Amérique ont à leur tour réagi et revendiqué le droit de vote. Si les travaux sur le suffrage féminin et les mouvements féministes dans l'Hexagone ont fait leur chemin ${ }^{1}$, en revanche, nous connaissons moins bien la lutte qu'ont menée les femmes des Antilles françaises pour leur intégration citoyenne ${ }^{2}$. Ces travaux n'ont d'ailleurs accordé que peu ou pas d'intérêt aux femmes des colonies françaises, supposant que ces dernières avaient obtenu le droit de vote sans grande bataille et que leurs plaidoyers rejoignaient, en partie, ceux des femmes métropolitaines. Cependant, leur combat était tout à fait singulier, compte tenu de leur statut dans le contexte colonial. Juridiquement, et jusqu'à l'ordonnance du 21 avril 1944, date à laquelle le droit de vote et d'éligibilité leur est accordé, les femmes, aussi bien en France que dans les colonies, ne jouissaient d'aucun droit civique et politique.

La question du suffrage féminin, évoquée timidement à travers les publications ${ }^{3}$ des premières décennies du XXe siècle, marqua un tournant dans la conception de la place de la femme dans la société antillaise. C'est une période où les femmes étaient au cœur de la question sociale. A travers les journaux, on les exhortait à participer à la régénérescence de la société, tout en conservant leur rôle traditionnel de bonne mère et d'épouse. Certaines profitèrent donc de cet espace, pour en conquérir d'autres et amorcer les premières revendications concernant le droit de vote.

\footnotetext{
${ }^{1}$ Dans les principaux ouvrages sur le féminisme français, aucune mention n'est faite quant à la lutte des femmes des colonies françaises d'Amérique. Citons quelques-uns : L'histoire des femmes en revues : France/Europe, Toulouse, Presses Universitaires du Mirail, 2002 ; Christine BARD, Les filles de Marianne, Histoire des féminismes (1914-1940), Paris, Fayard, 1995 ; Albert et Nicole DU ROY, Citoyennes. Il y a 50 ans le vote des femmes, Paris, Flammarion, 1994 ; Laurence KLEJMAN et Florence ROCHEFORT, L'égalité en marche. Le Féminisme sous la Troisième République, Paris, Presses de la Fondation Nationale des Sciences Politiques- Des femmes, 1989 ; Maïté ALBISTUR et Daniel ARMOGATHE, Histoire du féminisme français : du moyen âge à nos jours, Paris, Editions Des Femmes, 1977.

${ }^{2}$ Malgré de précieuses contributions : Arlette GAUTIER, «Femmes et colonialisme », Le livre noir du colonialisme, $X V I^{e}-X X I^{e}$ siècle : de l'extermination à la repentance, Marc FERRO (dir.), Paris, Robert Laffont, 2003, pp. 569-607 ; Myriam COTTIAS, «Mariage et citoyenneté dans les Antilles françaises (XVII-XIX) : de l'esclavage à la femme «poto-mitan », Mélanges à Jacques Adélä̈de Merlande, Danielle BÉGOT et Jean-Pierre SAINTON (dir.) Paris, Editions du CTHS, 2002, pp. 319-334; Myriam COTTIAS, «Gender and citizenship in French West Indies, 1848 1945", Slavery and abolition, 26, 1995, pp. 231-243.

${ }^{3}$ En Guadeloupe, on peut citer L'Emancipation et Le Colonial ; et en Martinique, La France Coloniale.
} 
Ainsi, à travers leur implication sociale, elles investirent des domaines qui traditionnellement étaient réservés aux hommes. Aux associations de secours mutuel traditionnelles ${ }^{4}$, dirigées par des hommes, viennent s'ajouter, au début du XXe siècle, de nouvelles sociétés dirigées et organisées par des femmes. Ces sociétés vont progressivement, et à des degrés différents, s'engager dans les débats publics, en réclamant l'amélioration des conditions sociales et juridiques des femmes. Le droit de vote, s'il ne constitue pas au début un enjeu majeur, donne lieu à des débats tant au sein de ces associations qu'au sein de la société. Cette émergence dans l'espace public va de pair avec les théories du moment qui postulent pour l'instruction laïque des filles et leur préparation à leur futur rôle de mère et d'éducatrice.

L'émergence des femmes dans l'espace public, à travers les sociétés de prévoyance, est cependant circonscrite au cercle restreint des femmes instruites, faisant partie de l'élite de couleur. Ces femmes de l'élite, véritables dames patronnesses, se donnaient pour mission d'aider les femmes, les enfants et les personnes désœuvrées, et leur mission philanthropique était à la mesure des difficultés que rencontraient les femmes dans la société. Elles occupaient une place privilégiée, par rapport aux autres femmes.

A l'époque, l'accès des femmes à certains métiers était encore limité. Dans les campagnes, elles représentaient un pourcentage élevé parmi les travailleurs agricoles, employées pour le sarclage, la culture et la coupe de cannes. Dans les villes, elles s'adonnaient aux travaux domestiques, à la couture, qui restait un moyen assez privilégié de travail indépendant, et commençaient à s'insérer lentement dans le système éducatif. Là où on les retrouve en grand nombre (l'agriculture), elles étaient moins bien rémunérées que les hommes. Certaines protestaient contre leur exploitation, comme l'indique l'enquête réalisée par Salinière ${ }^{5}$, en avril 1910, où certaines femmes dénoncèrent l'inégalité des salaires entre hommes et femmes, alléguant qu'elles travaillaient autant que les hommes.

[Dans la section des Mangles] Il y a lieu de signaler la protestation des ouvrières agricoles contre l'inégalité des salaires. Elles réclament 1f 50 pour la journée de travail. Elles déclarent qu'elles travaillent autant que les hommes. ${ }^{6}$

Ce sont ces conditions de vie précaires qui poussèrent la classe laborieuse des îles à développer des stratégies pour parer à l'infortune, aux frais occasionnés par un décès, une maladie, etc., en fondant des sociétés de prévoyance et de secours mutuel. L'analyse du tissu associatif féminin -les sociétés féminines d'assistance, de prévoyance ou de secours mutuel, mais également les associations féminines ouvertement politiques- permet de remonter aux premières formes

\footnotetext{
${ }^{4}$ Notons que les confréries et mutuelles existaient depuis la période esclavagiste.

${ }^{5}$ SALINIĖRE, Origines et causes du mouvement gréviste du mois de février 1910. Les petits planteurs (réclamations et incidents divers), Basse-Terre, Imprimerie du gouvernement, 1910.

${ }^{6}$ Ibid., p. 52.
} 
d'organisation des femmes antillaises dans le domaine civique. Ces sociétés mutualistes étaient, en effet, l'espace privilégié de l'expression et de l'intervention des femmes dans la vie de la cité. Ces sociétés, ont-elles servi de tremplin aux femmes pour conquérir d'autres espaces, notamment leur intégration dans des partis politiques ? Comment se font les premières revendications pour le suffrage féminin dans les premières décennies du $\mathrm{XX}^{\mathrm{e}}$ siècle et quels en sont les échos dans la société ?

Nous nous proposons donc d'analyser, pour les deux îles, la particularité des sociétés féminines de secours et de prévoyance, leur rapport avec la politique et leur continuité ou non dans des formes d'organisations plus politisées. Nous examinerons le biais par lequel les femmes guadeloupéennes faisaient entendre leurs revendications pour le suffrage, notamment à travers des journaux féministes 1'Echo de Pointe-à-Pitre (1918-1921), dont la première expérience éditoriale remonte à 1911, sous le nom de Pointe-à-Pitre (1911-1912) et leurs échos dans la société de l'époque.

\section{Les sociétés mutuelles et les solidarités politiques}

Les premières sociétés féminines d'assistance et de secours mutuel naissent en Guadeloupe dès la fin du $\mathrm{XIX}^{\mathrm{e}}$ siècle. La Mutuelle des ménagères ${ }^{7}$ et les Roses fanées ou Fleurs fanées, une association de «vieilles filles ${ }^{8}$ créée en 1884 et organisée en mutualité dès 1907, en sont des exemples. Les sociétés féminines sont fondées plus tardivement en Martinique, même s'il est à noter l'existence de l'Ouvroir des jeunes filles de Fort-de-France, institution créée dès 1825 pour accueillir les orphelines et les jeunes filles de la population ouvrière de Fort-de-France et dont la mission était «d'inculquer aux jeunes filles de la population ouvrière de Fort-de-France, par une éducation appropriée à leur condition, des habitudes d'ordre, de travail et de régularité. [...] Destinées à embrasser plus tard les professions d'ouvrières, de bonnes d'enfants, de domestiques, les jeunes filles admises dans cet établissement étaient principalement affectées à des travaux d'aiguille, aux soins du ménage, à la cuisine, à la buanderie et au repassage. Une heure seulement le matin et l'après-midi est consacrée à l'instruction religieuse et à la lecture » ${ }^{9}$. Les femmes faisaient déjà partie de bon nombre de sociétés de type professionnel -pour la plupart mixtes-, qui s'apparentaient aux confréries noires de l'époque esclavagiste ${ }^{10}$.

\footnotetext{
${ }^{7}$ La référence à la mutuelle des ménagères est donnée par Michel LEIRIS, Race et société. Contacts de civilisations en Martinique et en Guadeloupe, Paris, Les presses de l'UNESCO-Gallimard, 1974, p. 62.

${ }^{8}$ Ibid. Michel LEIRIS, dans son enquête, souligne que cette mutuelle féminine «présente ouvertement le caractère d'une société d'âge et pas seulement de sexe : les Roses fanées, [...] dont les adhérentes sont des vieilles filles ou des femmes âgées vivant sans homme ». Michel LEIRIS, Race et société..., p. 62.

${ }^{9}$ La terre des gens sans terre. Petite histoire de l'école à la Martinique (1635-1982), (Joseph JOS, dir.), Association des Membres de l'ordre des palmes académiques - Martinique, L'Harmattan, 2003, p. 83.

10 Jean-Pierre SAINTON, Les nègres en politique. Couleur, identités et stratégies de pouvoir en Guadeloupe au tournant du siècle, t.1, Thèse de doctorat, Université de Provence, Aix-Marseille 1, 1997, p. 149.
} 
L'analyse des premières sociétés féminines du début du siècle montre cependant un certain contraste entre la Guadeloupe et la Martinique. En effet, à la différence de la Guadeloupe où certaines sociétés féminines du début $\mathrm{du} \mathrm{XX}^{\mathrm{e}}$ siècle naissent dans la mouvance du parti socialiste, les sociétés féminines de la Martinique semblent à première vue apolitiques. Les sociétés féminines de la Guadeloupe, même si elles opèrent également dans la sphère sociale, sont politisées et rattachées en général au parti socialiste ou à la Libre Pensée : la Société des Vraies Filles de Schoelcher, société féminine de Libre Pensée ; les Vraies Filles de l'Espérance ; les Vraies Filles de l'Avenir (jeunes filles socialistes) ; la Société des Filles de la Victoire, créée en 1902 et qui comptait 46 membres en 1903 ; les Dames de Saint-Louis, dont la présidente en 1919 était Madame Ursen Boye $^{11}$; ou encore au parti radical républicain : c'est le cas de la société mutuelle féminine, le Sou des Dames, fondée en 1905 et qui déclarait 800 sociétaires en $1910^{12}$. En dehors de ces sociétés féminines, il existait une association féminine ouvertement politique, le Groupe des Femmes Socialistes, créée en 1902 et qui rassemblait le Groupe des Jeunes filles Socialistes et d'autres associations féminines de prévoyance qui y adhéraient.

Le parti socialiste et la Libre pensée ont favorisé l'intégration des femmes dans leurs rangs. Dans leur projet de «régénération » de la société, la femme avait un rôle incontournable. La Société des Vraies filles de Schoelcher (dont la présidente était Amélie Césaire) avait même une martyre : Armantine, fille du peuple abattue par la gendarmerie le 21 janvier 1892 et à qui elles rendaient hommage chaque année par «un pèlerinage de piété socialiste ${ }^{13}$. Ses membres effectuaient chaque 24 décembre un pèlerinage au musée Schoelcher, pour rendre hommage à son fondateur ${ }^{14}$. La Société des Filles de la Victoire, quant à elle, participait à toutes les manifestations du parti socialiste et se chargeait d'organiser la fête des Enfants de Marianne. La réorganisation sociale postulée par le parti socialiste prétendait embrasser toutes les composantes de la société.

En Martinique, il existait une société franc-maçonne, $L$ 'émancipation féminine ${ }^{15}$, non déclarée et affiliée à la Fédération française de l'ordre mixte international, «le Droit Humain ». Dans une conférence donnée au local maçonnique, le $1^{\mathrm{er}}$ mars 1931, Madame Olympe Claude, maîtresse de français au Pensionnat colonial et membre de la Loge, s'interrogeait : «Et pourquoi des ignorants, des ivrognes, des malades, des incapables, sont-ils électeurs et éligibles, alors que tant de femmes,

\footnotetext{
11 Information tirée de L'Echo de Pointe-à-Pitre, 30 août 1919, 30 édition. Archives départementales de la Guadeloupe.

${ }^{12}$ Ibid, SAINTON, p. 385.

${ }^{13}$ Archives départementales de la Guadeloupe. L'Emancipation, vendredi 5 février $1904, \mathrm{n}^{\circ} 102$.

${ }^{14}$ L'Emancipation, vendredi 25 décembre $1903, \mathrm{n}^{\circ} 96$.

15 Elle a été fondée en 1930 et dissoute par décret du 19 août 1940. Nous savons très peu sur l'organisation et la composition de cette société. Archives Départementales de la Martinique, Associations diverses (1919-1940).
} 
distinguées sont systématiquement écartées ?"16 En France, le Droit Humain réclamait le suffrage féminin, ainsi que la création en 1894 d'un groupe parlementaire des droits de la femme ${ }^{17}$. En dehors de cette société secrète, les associations d'assistance mutuelle en Martinique étaient très actives dans la sphère sociale, mais ne faisaient partie d'aucune formation politique : Saint-Louis des Dames et Prévoyance des femmes, toutes deux fondées en 1901 ; l'Union des Dames de Tivoli fondée en 1907 ; l'Union des Dames martiniquaises fondée en 1915 ; Les Guides de France, société féminine d'éducation physique fondée en 1928 et appartenant à la branche catholique des Scouts de France ; l'association des Dames Françaises de la Croix Rouge fondée en 1932.

Ces sociétés mutualistes féminines étaient fondées et gérées par des femmes de couleur de la bourgeoisie de Fort-de-France. Louise Nardal, Emilie Milienne, Louise Saint-Cyr et Gabrielle Sévère, pour n'en citer que quelques-unes, prirent une part très active dans les œuvres de bienfaisance et le secours aux nécessiteux. Ces femmes possédaient tout un réseau de solidarités horizontales et transversales et menaient un combat de front pour améliorer le sort des déshérités, s'appuyant sur leur position sociale et l'influence de leur mari. Ces mêmes femmes étaient membres actifs ou à la tête de plusieurs sociétés ${ }^{18}$. Leurs actions ne visaient donc pas uniquement l'amélioration des conditions de vie des femmes, mais touchaient également l'enfance abandonnée, les orphelins, les prisonniers et les jeunes incarcérés. Elles fondèrent, présidèrent ou soutinrent de nombreuses auvres caritatives : le Foyer des Orphelins de la guerre (1915), la Protection de l'Enfance Coupable, l'Oeuvre des Prisons (1927), le Vestiaire des Pauvres à Fort-de-France, l'Asile Bethléem, la Crèche de l'Union au quartier ouvrier des Terres Sainville, l'Ouvroir, etc.

Ces sociétés de secours mutuel ne formulaient aucune revendication sur le suffrage féminin, mais ont permis de donner une visibilité publique à la femme dans des domaines, comme les œuvres sociales, qui étaient conformes aux rôles que la société leur assignait. Comme le rappelait en 1914 C. Moynac, censeur proviseur du Lycée Carnot, durant le discours d'usage des distributions de prix au cours secondaire de jeunes filles à Pointe-à-Pitre, l'œuvre mutualiste a été très utile à la cause féminine, car selon lui, la femme s'est retrouvée sur le même pied d'égalité que l'homme :

\footnotetext{
16 Bibliothèque Nationale de France. L’Emancipation féminine et le droit humain. Conférence faite au local maçonnique le dimanche $1^{\text {er }}$ mars 1931 par Madame Olympe Claude, maitresse de français au Pensionnat colonial (membre de la Loge), Fort-de-France, Imprimerie R. Illemay.

${ }_{17}^{17}$ Raymond HUARD, Le suffrage universel en France, 1848-1946, Paris, Aubier, 1991, p. 193.

${ }^{18}$ Le 21 juin 1901, Louise Nardal fondait avec Gabrielle Sévère et Emilie Milienne, la Société féminine Saint-Louis des Dames. Gabrielle Sévère créait en 1914 l'Union des Dames Martiniquaises, Louise Nardal en était la Vice-présidente et Louise Saint-Cyr, la trésorière adjointe. Sous l'initiative de Gabrielle Sévère, elles fondaient ensemble la Croix-Rouge en 1932, qui résultait de la fusion de différentes sociétés des Dames Françaises. Victor Sévère, le mari de Gabrielle, était désigné en tant que membre du comité protecteur des Guides de France. Voir Monette CARÊME-LIÉNAFA, Femme et mutualité à la Martinique, 1893-1993. Ouvrage historique et mutualiste, 1994.
} 
«[...] Ces efforts porteront leurs fruits, plus heureuses que vos aînées vous vivrez dans une humanité plus équitable parce qu'elle devient chaque jour plus éclairée et moins superstitieuse. Vous aussi peut-être vous vous mêlerez à la vie sociale pour l'améliorer. Sans rien présager d'un avenir incertain, mais qui est tout proche et que vous verrez sûrement, sans donner des conseils que l'expérience risquerait de démentir, je crois pouvoir dire que le siècle qui s'ouvre sera pour la femme celui de la justice et de la liberté. Il dépend de vous de hâter son avènement, en sortant de cette réserve, de cette abdication de soi-même, de ces ruses innocentes qui furent la diplomatie de la faiblesse féminine. » 19

En ce qui concerne la composante ethnique et sociale de ces sociétés, un compte-rendu de 1913 sur l'assemblée annuelle de la fédération mutualiste de la Guadeloupe ${ }^{20}$, insistait sur la «solidarité étroite qui unit la corporation mutualiste toute entière. Intellectuels, négociants, ouvriers, tous liés par la plus franche cordialité, malgré les différences ethniques et politiques inévitables et nécessaires, communièrent intimement dans ce repas fraternel... ${ }^{21}$. Par rapport aux principes égalitaires dont elles s'inspiraient, s'y côtoyaient donc des individus de classes, de races et de couleurs politiques différentes. Ces sociétés mutualistes étaient le reflet de solidarités, non seulement sur le plan social mais également au niveau politique.

Il y a donc un lien étroit entre ces sociétés et le monde politique. Les sociétés féminines étaient dirigées en général par l'élite urbaine de couleur qui prenait une part active dans la politique. Les époux des dirigeantes de la société guadeloupéenne Le Sou des Dames, mesdames Bloncourt, Michineau, Choulon et Lafontaine, étaient des mulâtres, partisans d'Achille René Boisneuf, et à la tête de la fédération mutualiste. Certains, comme le socialiste Gaston Erlace, qui fut secrétaire général de la Fédération Mutualiste de la Guadeloupe ${ }^{22}$ et qui, en 1901, avait dirigé le journal $L a$ Crucifiée (novembre 1901- juin 1902) aux côtés du leader socialiste Légitimus ${ }^{23}$, s'était érigé en défenseur de la cause féminine et en mécène. La société des Fleurs Fanées naquit ainsi sous sa présidence ${ }^{24}$. C'est encore lui qui incita, en 1919, les membres de la société des Dames de SaintLouis à s'organiser, afin de réclamer leurs droits politiques. Ces sociétés féminines qui avaient démontré leur modération, leur dynamisme et leur respectabilité, étaient convoitées par les partis politiques, du fait du public qu'elles attiraient lors de leurs manifestations culturelles (fête des fleurs, soirées de gala, causeries, etc.) et servaient de vitrine de l'intérêt que les partis politiques accordaient à la question féminine.

En Martinique, même si les sociétés féminines n'étaient pas ouvertement politiques, les époux de leurs dirigeantes étaient des personnages importants de la vie politique. Victor Sévère, le mari de

\footnotetext{
${ }^{19}$ Archives départementales de la Guadeloupe. Cours secondaires de jeunes filles, Pointe-à-Pitre. Distribution solennelle des prix, sous la présidence de Monsieur Jocelyn Robert. Le discours d'usage a été prononcé par Monsieur Ch. Moynac, chevalier de la légion d'honneur. Censeur p. du Lycée Carnot, 1914. Imprimerie commerciale, 13 rue d'Arbaud.

${ }^{20}$ Cette fédération regroupait la majorité des sociétés de prévoyance.

${ }^{21}$ Archives départementales de la Guadeloupe. Le Colonial, mercredi 9 juillet 1913. Mutualité, solidarité, fraternité.

${ }^{22}$ Archives départementales de la Guadeloupe. L'Echo de Pointe-à-Pitre, 30 octobre 1918.

${ }^{23}$ Op. cit., Jean-Pierre SAINTON, planche 45, p. 438 a, b.

${ }^{24}$ Archives départementales de la Guadeloupe. L'Echo de Pointe-à-Pitre, 30 août 1919.
} 
Gabrielle Sévère, était avocat et homme politique très en vue en Martinique : élu maire de Fort-deFrance en 1900, il devint conseiller général de cette ville et député du nord de la Martinique. Dès les années 1907, il fonda un journal, La France coloniale, organe de la démocratie Martiniquaise, qui publiait quelques articles sur l'émancipation des femmes. Victor Sévère était en relation avec le mari de Louise Nardal, l'ingénieur Paul Nardal, qui écrivit même un article dans la colonne de son journal. Sous le mandat de Victor Sévère, le frère d'Emilie Etienne était conseiller municipal. Ces solidarités politiques avaient bien entendu des incidences sur les sociétés mutuelles féminines. En 1914, le comité d'assistance aux blessés de 1'Union des dames martiniquaises était géré par les épouses des personnalités politiques les plus importantes de l'île, entre autres mesdames Richard, femme du lieutenant-colonel et Commandant supérieur des troupes, Knight (dont le mari était sénateur et fondateur avec Lagrosillière du journal Le Bloc) et Lagrosillière ${ }^{25}$.

\section{Le Groupe des Femmes Socialistes et la position du parti socialiste guadeloupéen face au suffrage féminin}

L'Emancipation, organe du parti socialiste guadeloupéen, annonçait les réunions et les diverses manifestations organisées par le groupe des femmes socialistes de Pointe-à-Pitre. Ces réunions se tenaient en général «tous les dimanches, à 3 heures de l'après-midi au siège social du groupe, 7 faubourg Henri IV. Les demandes d'admission et les correspondances, devaient être adressées à la citoyenne Antonia Erlace ». ${ }^{26}$ Antonia Erlace était probablement l'épouse ou la parente du précité Gaston Erlace. L'encadrement des militantes dans des structures politiques explique-t-il la précocité des revendications des Guadeloupéennes (par rapport à celles des Martiniquaises) pour le suffrage féminin ?

Les hommes politiques ont souvent mentionné la participation -quoique passive- des femmes antillaises à la politique. C'est ainsi qu'en 1919, le socialiste Gaston Erlace soutenait que les femmes, en dilettantes, prenaient part depuis longtemps à la politique et avaient pris «l'habitude de s'inféoder aux luttes politiques ». S'inspirant de la phrase d'un journaliste, ce dernier déclarait: «Aux colonies, sans la femme, il n'y a pas de politique $»^{27}$ L'illusion de ce pouvoir cachait une réalité accablante : la femme ne jouissait ni de droits civiques ni de droits politiques. Jean-Pierre Sainton a déjà rappelé la place que le mouvement socialiste guadeloupéen accordait aux femmes au début du $\mathrm{XX}^{\mathrm{e}}$ siècle :

«Les structures politiques leur sont ouvertes, y compris les congrès de la Fédération socialiste, ainsi que les organes dirigeants. Elles sont appelées à participer aux réunions des associations syndicales, des sociétés

\footnotetext{
25 Archives départementales de la Martinique. L'Union Sociale, vendredi 14 août 1914. L'union des Dames martiniquaises. Comite d'assistance aux blessés.

${ }^{26}$ L'Emancipation, vendredi 3 janvier 1903.

${ }^{27}$ L'Echo de Pointe-à-Pitre, 30 août $1919,30^{\mathrm{e}}$ édition.
} 
fraternelles et de libre pensée, ainsi qu'à celles des comités politiques et des assemblées électorales ; elles signent des articles dans la presse socialiste, diffusent le journal, interviennent dans les conférences et surtout président les fêtes et manifestations du mouvement.» ${ }^{28}$

En effet, l'inclusion des femmes dans ce parti marque la première participation active des femmes guadeloupéennes à la politique. En analysant le discours de ce parti sur le suffrage féminin, une certaine réticence à ce sujet est cependant perceptible et reflète en général la réserve du parti socialiste à l'égard du vote féminin. Dans sa mission de façonner la femme moderne, l'Emancipation abordait néanmoins la question du droit de vote, même s'il soumettait ce droit à l'instruction des femmes :

«Il reste à l'homme qu'un domaine dans lequel il se complait et qu'il invoque à chaque instant pour essayer de
justifier sa prétendue supériorité : c'est le droit électoral. Mais quand, dit une femme célèbre « On n'a su faire
d'autre besogne que celle dont vous avez doté le monde depuis le temps que vous le gouvernez; quand on n'a su
apporter le bonheur d'une partie de l'humanité qu'en asservissant l'autre, on n'a pas le droit de se montrer si fier,
si arrogant dans la conservation d'un droit dont on a fait un si mauvais usage.» 29

Devant les propos enthousiastes de la citoyenne Bonnevial ${ }^{30}$, qui assista en 1904 au congrès de la Libre Pensée à Rome sur l'émancipation morale et intellectuelle de la femme, le journal l'Emancipation reconnaissait la part immense de la femme dans la société moderne, la nécessité que son instruction soit égale à celle de l'homme et son accès progressif à des métiers jusque-là réservés aux hommes. Il fustigeait néanmoins les idées féministes et dénonçait les dérives de la lutte des sexes et du bouleversement des rôles :

«[...] Nous aurions alors assez souvent ce singulier spectacle de voir Monsieur tricotant des bas de laine tandis
que Madame, son quotidien en main discutera les actes ministériels, émettra son opinion sur la politique générale
et se décidera même pour faire échec au gouvernement, à briguer les suffrages de ses concitoyennes aux
élections législatives et sénatoriales. Car madame, ayant acquis son droit de vote, deviendra influente dans sa
région, se mettra à la tête des mouvements politiques et voudra diriger les affaires du pays, peut-être avec
modestie, peut-être avec autorité, peut-être même avec tambour et trompette. Cela ne nous offusquerait pas.
Cependant, je me crois permis de dire que ce ne serait certainement pas un idéal pour la femme moderne. Nous
autres socialistes, nous voulons l'émancipation de la classe prolétarienne, son bonheur moral et intellectuel sans
distinction de race ni de sexe. » 31

Les socialistes n'étaient pas unanimes quant à l'octroi du droit de vote aux femmes. Par conséquent, malgré leur soutien, la bataille n'était pas gagnée. En France également, à cette époque, les idées féministes étaient minoritaires (même parmi les femmes) et le suffragisme restait marginal dans le mouvement féministe. Aux Antilles, si l'idée de l'instruction égale entre hommes et femmes et l'accès des femmes dans certaines professions commençaient à se concevoir, il n'en était pas de même pour le suffrage féminin.

\footnotetext{
${ }^{28}$ Op. cit., Jean-Pierre SAINTON, p. 316.

${ }^{29}$ L'Emancipation, vendredi 17 juillet 1903. N ${ }^{\circ} 73$. La femme. Son passé, son présent, son avenir.

${ }^{30}$ Article paru dans La Petite République Socialiste et cité dans L'Emancipation, 4 novembre 1904.

${ }^{31}$ L'Emancipation, 4 novembre 1904. La femme moderne.
} 


\section{Pointe-à-Pitre et l'Echo de Pointe-à-Pitre et les premiers germes du féminisme militant en}

\section{Guadeloupe}

Il existe une certaine continuité entre les sociétés mutuelles féminines et les premières formes d'organisation politique des femmes. En Guadeloupe, ce lien est à chercher en la personne du socialiste Gaston Erlace, président de la fédération mutualiste et fondateur des journaux Pointe-àPitre et l'Echo de Pointe-à-Pitre. De quelle manière ces journaux féministes participaient-ils au débat sur le suffrage féminin ? Pointe-à-Pitre, organe des Dames des colonies (1911-1912), dont peu de numéros ont été conservés, était créé le 20 novembre 1911 et paraissait les 10, 20 et 30 de chaque mois. Sa devise était rappelée dès la première page : «La femme est un être divin, défendre ses intérêts, lui faire connaître sa valeur, c'est défendre l'humanité ». La direction et la rédaction de Pointe-à-Pitre étaient confiées à madame Marie Paule et à la veuve Th. Pierre, avec la collaboration des demoiselles Christine Germaine, Augustine Jean-Baptiste, Victoire Lazare, Clémence André, Noémie Charlemagne et Dame Tongalacer. Ces femmes étaient issues de la bourgeoisie de couleur de Pointe-à-Pitre et des communes.

Ce journal s'adressait avant tout à un public féminin, acceptant «tous les écrits qui pourront occuper les loisirs de nos Dames et de nos jeunes filles, tels : chansons, poésies, etc.». Au contraire des journaux essentiellement politisés, ces femmes rendaient visibles leurs préoccupations domestiques, leurs activités sur leur lieu de travail et dans leur vie sociale. Elles publiaient ainsi de nombreux articles sur la cuisine, donnaient des conseils sur le traitement pour la chute de cheveux, sur l'économie du ménage, sur la moralité des femmes, sur le comportement des hommes irrespectueux, et dénonçaient l'exploitation des apprenties couturières. Au début, l'idée d'une émancipation féminine n'était pas vraiment évoquée, et certaines collaboratrices rappelaient le rôle de la femme, le cantonnant à la sphère privée : épouse, mère et maîtresse de maison. D'autres articles, en revanche, abordaient la question du droit de vote féminin avec beaucoup d'optimisme, saluant les avancées dans ce domaine :

«Paris, 6 décembre. La commission électorale dont M. Brisson est président a adressé à la chambre un rapport
favorable sur le droit de vote des femmes en faveur des candidats aux Conseils généraux et municipaux et sur
l'éligibilité du sexe féminin à ces postes. Bravo!
Nous marchons donc vers notre émancipation et saurons le moment venu donner la preuve de notre intégrité. » 32

Après un an d'existence, c'est avec amertume que la directrice constatait que le public féminin ne soutenait pas leur cause, ne serait-ce qu'en s'acquittant du paiement de l'abonnement mensuel. Elle rappelait la mission du journal: le travail de défense morale, de préparation de l'émancipation féminine et de dénonciation des abus contre les femmes.

Notre journal s'adresse tout particulièrement à la femme à qui il appartient à chacune de prendre un abonnement puisqu'il vient prendre sa défense morale et préparer son émancipation.

\footnotetext{
${ }^{32}$ Bibliothèque nationale de France, Pointe-à-Pitre, numéro 7, 20 janvier 1912.
} 
[...] Cette feuille a été créée pour crier contre les abus et les iniquités dont nous femmes, sommes sans cesse la victime dans notre société coloniale. ${ }^{33}$

Consciente du vide juridique et de l'indifférence des lois visant à protéger la femme, considérée encore comme mineure, dame Th. Pierre dénonçait la domination masculine et voulait faire prendre conscience aux femmes que seule leur lutte pouvait changer cette situation.

\begin{abstract}
« Nous sommes courbées sous le joug avilissant et combien dégradant de l'homme qui exerce sur notre personne les plus cruelles abominations et sommes forcées -enfants dociles que nous sommes- de croiser les bras et de laisser faire. Si parmi nous il y en a qui goûtent au bonheur du ménage, nombreuses par contre sont celles dont la misère assiège et rapetisse. A qui nous plaindre ? A toutes nos souffrances morales la loi nous regarde, sourit et détourne la tête : ce sont des femmes ! Des enfants mineures, elles ne sont pas intéressantes, acclame-t-elle !

Donc c'est nous mêmes qui devons prendre notre défense. Ne sentons-nous donc pas qu'une pareille situation doit cesser et pour y parvenir faut-il au moins que nous nous réveillons pour faire entendre notre voix et notre protestation dans une feuille quelconque ?» 34
\end{abstract}

Elles suivaient l'actualité sur le droit de vote et d'éligibilité des femmes. Ce droit accordé aux femmes de Californie en 1911 ne passa pas inaperçu sous leur plume. Elles n'hésitaient pas à publier les opinions de certains personnages, tel Paul Bonnaves, hostile à l'idée d'accorder aux femmes les mêmes droits que les hommes, mais qui se définissait «féministe », dans le sens d'amant de la beauté féminine. Selon ce dernier, les femmes réclamaient avant tout « le droit de se conduire aussi mal que les hommes ». Suzanne Gall, l'une des féministes de première heure, répondait à ces arguments :

« Si la femme cherche à égaler l'homme et réclamer aujourd'hui ses droits, c'est qu'elle se croit en mesure de se conduire. [...] Si notre rôle consistait seulement à nous mal conduire, notre état d'infériorité ne nous aurait nullement préoccupées et les progrès du jour ne nous auraient pas touchées de si près. Quoi, vous voulez arrêter l'élan de toute une génération qui se réveille, qui est en marche?

Mais vous ne croyez pas que les éléments même du temps sont avec nous? Si vous ne voulez pas du progrès, vous aurez des révolutions.» 35

La substitution de Marie Paule par Suzanne Gall à la rédaction du journal voit le ton du journal se durcir contre les esprits «chimériques, susceptibles et incultes ». Cette dernière affichait sa ténacité dans la lutte pour la reconnaissance des droits civiques et politiques des femmes :

[...] jusqu'ici la question féministe n'a été qu'ébauchée à la Guadeloupe. Des esprits inféodés au mal, par une campagne sournoise ont dès la première heure travaillé à la discréditer, à faire le vide autour de nous : parce que Mademoiselle Paule tachait de corriger agréablement certains travers de la Société, certains vices qui forment l'apanage de certains caractères. [...] Je constate que la Société telle qu'elle est organisée politiquement et civilement parlant, demande à être modifiée afin de reconnaître à la femme les droits dont elle est privée jusqu'ici. Victime de l'injustice des inconscients, elle se plonge toujours rêveuse dans un coupable avilissement. $^{36}$

\footnotetext{
33 Ibid, numéro 8, 30 janvier 1912.

${ }^{34}$ Pointe-à-Pitre, numéro 8, 30 janvier 1912.

35 Ibid, numéro 10, 20 février 1912.

${ }^{36}$ Pointe-à-Pitre, numéro 11, 30 février 1912.
} 
C'est avec la même virulence qu'une autre collaboratrice, Mercedes, critiquait Ti-ben (pseudonyme donné à une personnalité importante de l'île), adversaire du féminisme, et s'insurgeait contre le rôle traditionnel des femmes :

\footnotetext{
« Il faut avouer que l'aimable M. Ti-ben n'est pas tendre pour la femme, du moins dans ses écrits. Il ne croit pas à son émancipation totale dans un temps rapproché ; c'est pour lui un rêve irréalisable. Accompagner l'homme dans la vie, allaiter, élever ses enfants, les consoler à l'heure des déceptions, de la désespérance, les encourager, les récompenser, à l'heure des succès heureux, voilà le rôle dans lequel il voudrait la voir se confiner.

Eh bien non Monsieur Ti ben ! Il n'en sera pas comme vous croyez. L'évolution de la femme est commencée, elle se poursuivra, quelles que soient les coalitions qui puissent se former pour l'arrêter. » ${ }^{37}$
}

Avec l'Echo de Pointe-à-Pitre, organe pour le développement du Féminisme et la défense des classes laborieuses (1918-1921), fondé en 1918, les revendications devinrent ouvertement féministes et les positions plus radicales. La position de ce journal féministe était très avant-gardiste pour l'époque, car il utilisait la métaphore de l'esclavage pour fustiger la société. Il affirmait que, malgré l'abolition de l'esclavage en 1848, la femme guadeloupéenne vivait encore dans un état d'esclavage. Ces propos avaient d'ailleurs une résonance toute particulière dans une population à peine sortie du joug de l'esclavage.

Ces revendications révèlent une prise de conscience toute nouvelle des femmes antillaises de la spécificité de leur lutte émancipatrice. Ainsi n’hésitaient-elles pas à interpeller les hommes, les obligeant à se rendre compte que la soumission de la femme antillaise était en tout similaire à leur condition servile aux temps de l'esclavage ${ }^{38}$. Elles dénonçaient la domination qu'elles subissaient de ces mêmes « dominés » qui furent un temps esclaves.

D'autres journaux de l'époque faisaient-ils échos de ces revendications ? Quelques femmes prenaient part discrètement à la rédaction d'articles dans certains journaux. Lulu, Anita, Renée, Rita et Julia publièrent quelques articles dans le journal indépendant, le Colonial, qui proposait en autres des articles, poèmes et témoignages sur la beauté de la femme. En 1911, dans un article intitulé « Mon carnet », un certain Mathurin s'exprimait dans le Colonial sur le droit politique des femmes : au début s'il reconnaissait la nécessité d'aborder la question des droits de la femme, de son accès à des métiers jadis réservés aux hommes, c'est avec une pointe d'ironie qu'il concevait l'égalité juridique des deux sexes :

«Il est donc naturel que la femme réclame ses droits et, puisque nous l'obligeons à gagner son pain, il n'y a plus de raison que nous lui refusions d'être représentée au parlement ou dans tous les endroits où seuls, jusqu'à pressent les hommes étaient admis. [...] Or donc, elle aura les mêmes droits que l'homme, par contre, elle devra logiquement supporter les mêmes charges, dès l'instant qu'elle est capable de faire les mêmes travaux que l'homme, de diriger sa vie sans ses conseils, il est incontestable qu'elle n'a plus besoin de sa protection et qu'en outre il ne saurait être astreint à subvenir à ses besoins. Par conséquent, voici venir une ère nouvelle. Ne

\footnotetext{
${ }^{37}$ Pointe-à-Pitre, numéro 11, 30 février 1912.

38 Clara Palmiste, «L'utilisation de la mémoire de l'esclavage dans les revendications des féministes Guadeloupéennes (1918-1921) », dans Colonialismes, Revue Sextant, n²5, Editions de 1’Université Libre de Bruxelles, 2008.
} 
désespérons pas, Messieurs, de nous voir bientôt travestis en bonnes d'enfants, à moins que d'ici là on ait créé une nursery nationale !» 39

Dans un autre article intitulé, «Féminisme », le Colonial relatait, avec une certaine ironie, les mésaventures de Marguerite Isnard, avocate à Avignon, dont la demande pour se présenter aux examens de la magistrature d'arrondissement comme juge suppléant avait été rejetée :

«Et voilà comment cette année encore, en France, l'émancipation féminine, tout en ayant fait un grand pas, ne verra point siéger, doublement en robe, une des plus combatives, mais aussi des plus agréables suffragettes. » 40

En Martinique, même si les femmes ne possédaient pas d'organe de presse, certains journaux diffusaient l'actualité sur le suffrage féminin. En 1912, la France Coloniale publiait un article sur l'élection d'une femme député en Autriche; en 1913 sur l'élection d'une femme sénateur dans l'Etat du Colorado ; et en 1914 un article au titre provocateur : Femmes ! Voulez-vous voter ? Aux urnes, citoyennes ${ }^{41}$ !, s'inspirant d'une expérience réalisée en France par le Journal, «dans le but de savoir si oui ou non les femmes veulent voter ». Cette expérience avait également pour but de savoir combien de femmes s'intéressaient à la politique, si leur vote apporterait des changements et surtout de répondre à l'un des arguments souvent mis en avant contre le vote féminin, c'est-à-dire «les Françaises ne tiennent pas à voter». La France Coloniale du 13 avril 1918 annonçait la création en France d'une revue féminine, l'Evolution Féminine, et publiait un article plutôt rassurant sur la position de ses collaboratrices :

« Ne vous méprenez pas sur le sens de ce titre. Il ne dissimule ni tendances subversives, ni étendard de révolte. Notre revue groupera des femmes parfaitement équilibrées qui sauraient s'entendre pour le triomphe de la cause commune. Tout esprit de parti, toute querelle religieuse, politique ou sociale seront vigoureusement bannis de nos rubriques.

[...] Donc si ce titre «L'évolution féminine », évoque une image en votre esprit, que ce soit celle de la féminité alliée au féminisme. [...] Et nul homme, si anti-féministe soit-il, n'osera nous ridiculiser ou seulement sourire en lisant notre devise : Soyons sensibles à la souffrance de tout ce qui souffre ici-bas ». ${ }^{42}$

Même si elles reconnaissaient que le sort de la femme devait être amélioré au niveau professionnel, légal et moral, les propos de ces femmes, soucieuses de ne pas choquer les hommes, étaient modérés. Elles entendaient réconcilier féminité et féminisme, car elles savaient que la satisfaction de leur revendication dépendait d'un pouvoir essentiellement masculin. D'autres journaux postérieurs, avec des tendances diverses, comme le Flambeau, organe de l'association panafricaine- section Martinique, opposé au suffrage universel, fustigeait, en 1924, le droit de vote aux femmes :

«Le suffrage universel tel qu'il est pratiqué en France et ailleurs, est la plus grande loufoquerie des temps modernes. [...] En effet, il est fortement question à la chambre d'accorder le droit de vote aux femmes,

\footnotetext{
${ }^{39}$ Le Colonial, 26 avril 1911.

${ }^{40}$ Le Colonial, 24 Mai 1911.

${ }^{41}$ Archives départementales de la Martinique. La France coloniale, samedi 18 avril 1914.

42 Archives départementales de la Martinique. La France coloniale, 13 avril 1918. L’évolution féminine.
} 
lesquelles ne les demandent d'ailleurs pas. Et quand je dis que la folie de ce suffrage égalitaire sera ainsi doublée, je suis au-dessous de la vérité, puisque en France, il y a beaucoup plus de femmes que d'hommes.

[...] Quand les femmes voteront, Madame Curie se croisera dans le même bureau de vote avec Zizi Monoeil, des promenoirs parisiens, son égal devant la loi. » ${ }^{43}$

La bataille n'était donc pas gagnée, même si la société prenait position sur la question de l'égalité politique. Pointe-à-Pitre et l'Echo de Pointe-à-Pitre subirent un véritable «boycottage », certains abonnés refusant même de payer leur abonnement. L'exemple d'un ancien notaire qui refusa l'abonnement à l'Echo l'illustre bien :-«Je ne veux pas de ce journal, ne continuez pas à me l'envoyer, car je ne suis pas féministe. Cette idée ne prendra jamais ici $»^{44}$. L'annonce du vote du projet de loi accordant le droit de vote et d'éligibilité à la femme par le parlement en mai 1919, provoqua un déferlement de propos anti-féministes. Comme le relata Dame Th. Pierre :

«L'éligibilité des femmes aux assemblées élues, déclarent-ils, c'est le désordre dans les ménages. C'est le trouble porté dans les unions, c'est du scandale, c'est de l'anarchie, c'est l'oligarchie dans la Démocratie, c'est l'abomination dans la désolation, cette loi a été votée par des fous.» 45

Les collaboratrices de l'Echo reprochaient à leurs homologues masculins leur obstination à vouloir perpétuer une situation qui, en réalité, les avantageait : «À vous donc toutes les faveurs, tous les plaisirs, toutes les douceurs, tous les privilèges, toutes les joies. À nous femmes, toutes les humiliations, toutes les atrocités, toutes les injustices, toutes les vexations, toutes les cruautés, n'est-ce pas ! ${ }^{46}$

\section{Conclusion}

La participation des femmes à l'œuvre mutualiste leur a permis de se rapprocher de la sphère politique, car sous leurs apparences d'œuvres sociales, ces sociétés féminines étaient liées directement ou indirectement au monde politique, par le biais de solidarités horizontales et transversales. Leur intégration à la politique, à des degrés différents en Guadeloupe et en Martinique, a favorisé une prise de conscience de la nécessité de réclamer leurs droits et souligne leur volonté de ne pas rester à l'écart des grands changements qui s'opéraient dans la condition féminine. La création de Pointe-à-Pitre et de l'Echo de Pointe-à-Pitre, marque leur première tentative de mettre au devant de la scène leurs préoccupations sociales et leurs revendications pour le suffrage. Le militantisme des femmes guadeloupéennes, dès le début du XXe siècle, a été en partie favorisé par le parti socialiste qui misait sur leur intégration dans ses rangs, pour contribuer au progrès social. Les femmes ont su en profiter et aller outre ces objectifs, en se positionnant par

\footnotetext{
43 Archives départementales de Martinique. Le Flambeau, organe de l'association panafricaine, section Martinique. 19 et 26 janvier 1924. A propos du vote des femmes.

${ }^{44}$ L'Echo de Pointe-à-Pitre, 20 avril $1919,17^{\mathrm{e}}$ édition.

${ }^{45}$ L'Echo de Pointe-à-Pitre, 30 mai, $1919,21^{\mathrm{e}}$ édition.

${ }^{46}$ Ibid., 30 mai, 1919, $21^{\mathrm{e}}$ édition.
} 
rapport à la société et en dénonçant leur condition d'esclave. Ces deux journaux restent donc exceptionnels, car il faudra attendre les années 40 pour que les femmes fondent des journaux analogues: Cendrillon (1947), organe mensuel de la section guadeloupéenne de l'Union des Femmes françaises ${ }^{47}$; La Femme dans la Cité (1945), organe du Rassemblement féminin de Martinique ; Femmes martiniquaises (1947), organe de l'Union des Femmes de la Martinique.

Il faudrait voir dans quelle mesure l'organisation des femmes au début du $\mathrm{XX}^{\mathrm{e}}$ siècle a servi de «moule» à leurs actions dans les années 40, car est-ce un hasard que deux femmes (Eugénie Eboué-Tell et Gerty Archimède) aient été élues en Guadeloupe députés à l'Assemblée Constituante en 1945 et en 1946 ? Dans le cas de la Martinique, la continuité des solidarités tissées dans les trois premières décennies du $\mathrm{XX}^{\mathrm{e}}$ siècle, à travers les œuvres sociales, est assurée, car Paulette Nardal, fille de Louise Nardal, sera à la tête du Rassemblement féminin, l'organisation féminine qui s'impliquera le plus dans les œuvres sociales.

\footnotetext{
${ }^{47}$ Elle deviendra en 1958, l'Union des Femmes Guadeloupéennes.
} 